\title{
Occurrence of Swine Salmonellosis in Postweaning Multisystemic Wasting Syndrome (PMWS) Affected Pigs Concurrently Infected with Porcine Reproduction and Respiratory Syndrome Virus (PRRSV)
}

\author{
Satoshi MURAKAMI ${ }^{1}$, Akihiro OGAWA ${ }^{2)}$, Tomohide KINOSHITA ${ }^{3)}$, Atsuko MATSUMOTO ${ }^{2)}$, Noriko ITO $^{2)}$ and \\ Takashi NAKANE ${ }^{4}$ \\ ${ }^{1)}$ Tokyo University of Agriculture, 1737 Funako, Atsugi 243-0034, ${ }^{2)}$ Hokubu Animal Health and Hygiene Service Center, Sawara, Chiba \\ 287-0004, ${ }^{3)}$ Toubu Animal Health and Hygiene Service Center, Tougane, Chiba 283-0064 and ${ }^{4)}$ Chiba Prefectural Livestock Research \\ Center, Yachimata, Chiba 289-1113, Japan
}

(Received 28 July 2005/Accepted 14 December 2005) ABSTRACT. Fourteen diseased pigs from four farms in which there had been an outbreak of salmonellosis were investigated. Granuloma-
tous inflammation with depletion of lymphocytes was observed in the swollen lymph nodes in these pigs. Antigens to porcine circovirus
type 2 (PCV2) were immunolabeled in the lesions along with detection of viral DNA as PCV2 by polymerase chain reaction (PCR). In
addition, antigens to porcine reproductive respiratory syndrome virus (PRRSV) were immunodetected in the lungs and Salmonella Chol-
eraesuis was isolated from the affected pigs. The nine salmonellosis affected pigs, five (55.6\%) with salmonellosis and PMWS concur-
rently infected with PRRSV were much higher than those infected with salmonellosis and PMWS (22.2\%) or with salmonellosis and
PPPRV $(22.2 \%)$.

KEY WORDS: PMWS, PRRSV, Salmonellosis.

In recent years, postweaning multisystemic wasting syndrome (PMWS) has been recognized as one of the most important contributors to swine production loss in the world. It has been strongly suggested that PMWS causes secondary immunodeficiency in pigs due to lymphopenia and severe damage to lymphoid tissues in the affected pigs [26]. Concurrent infectious viruses, such as Aujeszky's disease virus (ADV) [18], porcine parvovirus (PPV) [2], reproductive and respiratory syndrome virus (PRRSV) [19, 27], have all been isolated from PMWS affected pigs. Of these, it is known that pigs concurrently infected experimentally with PCV2 and PRRSV $[1,6,21]$ or PPV $[13,14]$ will show only PMWS lesions or clinical PMWS.

Apart from PMWS, in recent years, there has been anxiety concerning increased swine salmonellosis in Japan, particularly, Salmonella Choleraesuis infection following a massive outbreak of salmonellosis [23]. PRRSV exacerbates clinical signs of the disease in pigs [29, 31]. However, so far, the reason for the occurrence of a severe clinical disease in the field has not been sufficiently discussed. In the present investigation, we report on four pig farms which had experienced highly mortality due to $S$. Choleraesuis infection with PMWS, to ascertain whether there may be some relationship between the increasing causes and the occurrence of PMWS.

Between 1999 and 2002, on four integrated pig farms (A, $\mathrm{B}, \mathrm{C}$ and D) in Chiba, Japan, postweaning pigs approximately 40-90 days old showed clinical signs of progressive weight loss, anorexia, severe respiratory distress, cyanosis of both side of pinna and pyrexia over a period of several months. Diarrhea was not a common clinical sign. The diseased pigs from farms A and B were submitted to our labo- ratory several months after the outbreak. The pigs from farm $\mathrm{D}$ were submitted during the outbreak. Four diseased pigs from farm $\mathrm{C}$ were submitted in the course of the outbreak and two were sent at 4 months later.

The mortality rates of the pigs on the farms were A: $4.2 \%$, B: $11.0 \%$, C: $25.0 \%$ and D: $33.3 \%$, when the diseased pigs were first submitted. On those farms, each of the suckling and weanling pigs had been injected with inactivated vaccine against Mycoplasma hyopneumoniae. Three farms (A, $\mathrm{B}$ and $\mathrm{D}$ ) had used live vaccine against $\mathrm{ADV}$ in the weanling pigs, and farm $\mathrm{C}$ had used the vaccine in the suckling and weanling pigs. In farms $\mathrm{A}$ and $\mathrm{C}$, the postweaning pigs approximately 60-70 days old had also been injected with live vaccine against classical swine fever virus and Erysipelothrix rhusiopathiae. Farm D had injected live vaccine against PRRSV and E. rhusiopathiae to pigs at the same stage.

Fourteen diseased pigs obtained from 4 farms (A: 2, B: 3, C: 6 and D: 3 ) were sedated and anesthetized with azaperone and thiopental sodium, then killed by exsanguinations. At autopsy, the brain, heart, lungs, liver, spleen, kidneys, jejunum, ileum, colon and several lymph nodes including superficial inguinal, submandibular, mediastinal, and mesenteric lymph nodes were removed for bacteriological examination, and the remainder of these organs including the tonsils were fixed with $20 \%$ neutral buffered formalin. Fixed tissues were then embedded in paraffin, sectioned at 3 to $4 \mu \mathrm{m}$, and stained with haematoxylin and eosin (H\&E). The sections which bacteria were isolated from lung and liver were stained by Gram's Method.

Immunohistochemical detection of PCV2 in the sections containing PMWS lesions were performed by the same 
method as that described by Ounki et al. [16]. Sections were treated with $0.25 \%$ trypsin in order to activate the antigen. After the treated sections were applied with porcine normal serum (Dako Cytomation Co., Tokyo Japan), diluted $1: 10$, biotinylated anti-PCV porcine serum [16] that reacted with PCV2 but not PCV1, diluted 1:32, was used as the primary antibody. Controls for the immunoreactions were composed of specimens that did not contain primary antibodies. Then, the sections were immunostained with streptavidinbiotin-peroxidase (SAB) solution (Nichirei Inc., Tokyo Japan).

For the detection of PRRSV and Salmonella serovar O7 group antigens, the sections of lungs used for the detection of PRRSV antigens, as well as sections of lung and liver, besides, the sections of ileum and colon from 7 diseased pigs (C: 4 and D: 3 ) used for the detection of Salmonella serovar $\mathrm{O} 7$ group antigens were immunostained by means of SAB method. Rabbit antiserum against PRRSV [9], diluted 1:6,400, and Salmonella serovar O7 group (Denka Seiken Inc., Tokyo Japan), diluted 1:160, were used as primary antibodies. The sections for the detection of PRRSV antigen were treated with $0.25 \%$ trypsin except for the other sections for the detection of Salmonella serovar O7 group antigens. Controls for the immunoreactions were composed of specimens without primary antibodies and which were rinsed with normal rabbit serum (Nichirei Inc., Tokyo Japan). Each antigens-antibodies reaction was immunolabeled by applying Histofine SAB (R) Kit (Nichirei Inc., Tokyo Japan).

Samples from the brain, heart, lungs, liver, spleen, kidneys, jejunum, ileum, colon and lymph nodes were streaked onto desoxycholate-hydrogen sulphide-lactose (DHL) agar plates (Eiken Inc., Tokyo Japan) and horse blood agar plates. The plates were incubated at $37^{\circ} \mathrm{C}$ overnight. The colonies were isolated from the blood agar plates, and bacteriological species were identified with simple kits following api $20 \mathrm{E}$, (Nippon BioMérieux Tokyo Japan) and the rapid ID test HN-20 (Nihon Seiyaku Inc. Tokyo Japan). Salmonella like colonies isolated from DHL agar plates were examined biochemically for Salmonella, and confirmed serologically by the application of standard methods.

Viral DNA was extracted from the lungs, liver, spleen, kidneys, tonsils and inguinal, mesenteric and submandibular lymph nodes from seven diseased pigs (B: 3 and C: 4) with a commercial DNA isolation kit (SepaGene, Sanko Junyaku Inc., Tokyo Japan). For the detection of PCV, a species-specific primer pair (N3f: GTGAAGTGGTATTTGGGTGCCCGCTG and N4r: ATTGCTGGTAATCAAAATAC TGCGGGCC') which was designed by Hamel et al. [5] was used. The PCR amplified products were analyzed by gel electrophoresis and found to be an amplicon of about $550 \mathrm{bp}$ in size. Furthermore, these products digested with a restriction enzyme Hinfl into two fragments, $174 \mathrm{bp}$ and $378 \mathrm{bp}$, for $1 \mathrm{hr}$ at $37^{\circ} \mathrm{C}$ and the digested pattern was related to the PCV2 genome [5]. The products were analyzed by gel electrophoresis.

At autopsy, enlargement of superficial, mesenteric and tracheobronchial lymph nodes was commonly found in all affected pigs. Although there were some differences between lung lesions in the examined pigs, non-collapsed lungs with interstitial oedema, haemorrhages or tan mottling were observed. Furthermore, three (21.4\%) lungs (B: 2 and $\mathrm{C}: 1)$ showed mild to severe haemorrhagic consolidation together with hydrothorax and pleural adhesions. Three lungs from farm $\mathrm{C}$ and one set of lungs from farm $\mathrm{D}$ showed purulent and/or necrotising consolidation; of these, one contained disseminated nodules, which reached a maximum diameter of $3 \mathrm{~mm}$, in all lobules. In addition, there were scattered tiny necrotic and/or haemorrhagic foci in the liver in $7(50.0 \%)$ of these affected pigs.

In the microscopical findings, the most common lung lesions in the 14 diseased pigs were variable degrees of lymphohistocytic interstitial pneumonia, sometimes containing neutrophilc infiltration in the lesions. In alveolar and bronchial spaces, there were sometimes large numbers of neutrophils and/or coagulates of necrotic debris accompanying haemorrhages. Furthermore, additional lung lesions observed were suppurative necrotising bronchopneumonia $(21.4 \%)$, residual abscesses $(7.1 \%)$ and haemorrhagic fibrous pleuropenumonia $(21.4 \%)$. In the liver, so-called "paratyphoid" nodules or necrotic foci with haemorrhage and fibrous exudation were distributed randomly throughout the hepatic parenchyma in $9(64.3 \%)$ of the 14 diseased pigs. Gram-negative bacteria were often detected in the lesions. Furthermore, small focal lesions consisting of several apoptotic hepatocytes and slight infiltration of mononuclear cells were randomly seen in 10 cases. Apart from the hepatic lesions, one liver revealed histocytic or multinuclear giant cells infiltrations in the nodules analogous to "paratyphoid" nodules in addition to moderate perilobular fibrosis (Fig. 1a). Lymphoid organs including the lymph nodes, tonsils, spleen and Peyer's patches were found as PMWS lesions and consisted mainly of slight to moderate histocytic and/or multinucleate giant cells infiltration with variable degrees of lymphocytic depletion. In addition, basophilic intracytoplasmic botryoid inclusion bodies were frequently detected in the cytoplasm of histocytic or multinucleate giant cells in the lesions, and loss of the follicular architecture was often observed in the lymphoid tissues. Other lesions included slight meningoencephalitis $(14.3 \%)$, slight myocarditis (28.6\%), interstitial nephritis (78.6\%) accompanied by granulomatous lesions and neutrophilic and lymphohistocytic infiltration in lamina propria and submucosa of ileum and colon with desquamated epithelial cells (7.1\%).

Table 1 summarises the immunohistochemical detection of PCV2, PRRSV and Salmonella serovar O7 group antigens in examined tissues of the 14 diseases pigs. Positive antigens to PCV2 were immunolabeled in the granulomatous lesions in lymphoid tissues and kidneys. The labelling was most frequently seen in the basophilic intracytoplasmic inclusion bodies in the lesions. In the liver sections, there was the immunolabelling of hepatic nucleus in the small focal lesions. However, the labelling was not revealed in "paratyphoid" nodules or necrotic foci. Moreover, PCV2 


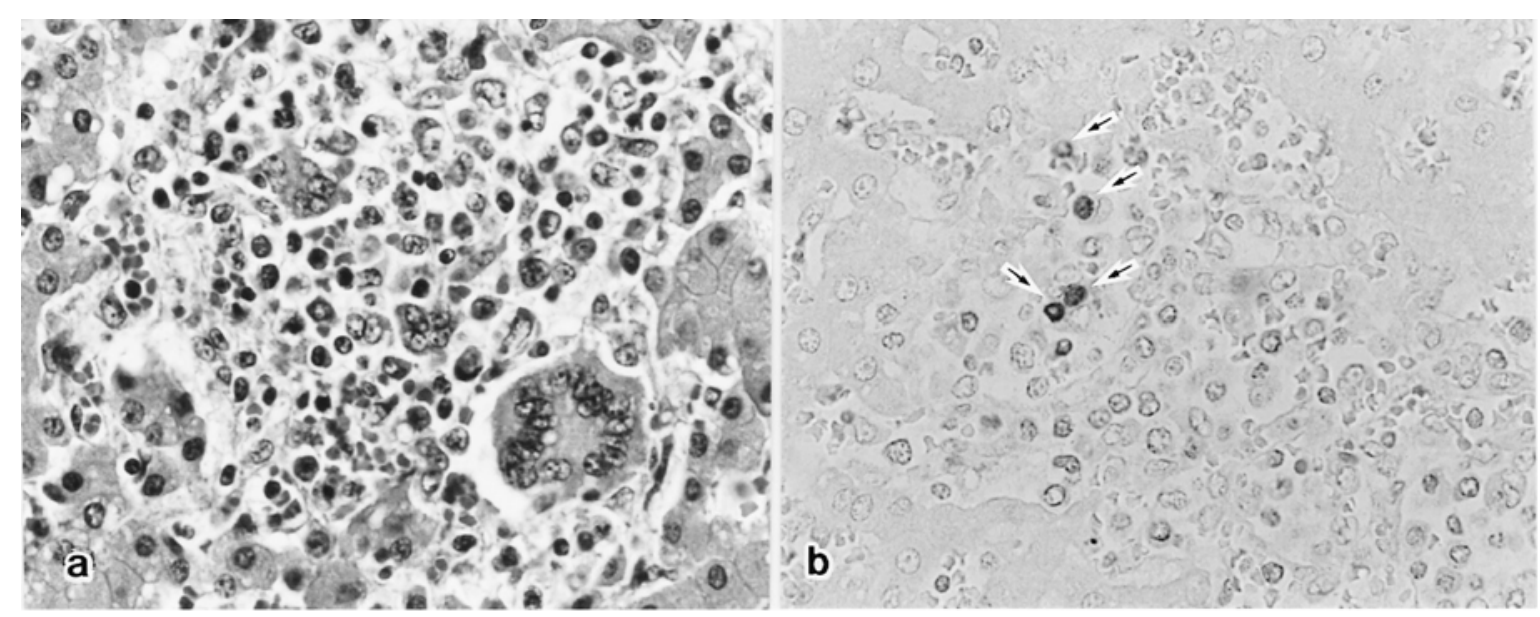

Fig. 1a. Liver: presence of histocytic and/or multinuclear giant cells in the nodules analogous to "paratyphoid" nodule. H\&E stain. $\times 400$. b. Positive immunoperoxidase reaction for PCV2 in the histocytic and/or multinuclear giant cells (arrows). SAB method. $\times 400$.

Table 1. Association between the diseases or lesions and immunohistochemical detection of Salmonella serovar O7 group, PRRSV and PCV2 antigens in the 14 diseased pigs

\begin{tabular}{|c|c|c|c|c|c|c|c|c|c|c|c|c|c|c|}
\hline Farm & & A & & B & & & & & $\mathrm{C}$ & & & & $\mathrm{D}$ & \\
\hline Pig No. & 594 & $595^{\S}$ & $593^{\S}$ & 600 & 601 & 381 & $382^{\S}$ & $387^{\S}$ & $388^{\S}$ & 578 & 579 & $350^{\S}$ & $351^{\S}$ & $352^{\S}$ \\
\hline Salmonellosis & $-/-*$ & $+/+$ & $+/+$ & $-1-$ & $-1-$ & $+/+a)$ & $+/+$ & $+/+$ & $+/+$ & $-1-$ & $-/+$ b) & $+/+c)$ & $+/+$ & $+/+$ \\
\hline Interstitial pneumonia & $+/ \mathrm{p}$ & $+/ p, s$ & $+/ \mathrm{s}$ & $+/ \mathrm{p}$ & $+/ \mathrm{p}$ & $+/ \mathrm{p}, \mathrm{s}$ & $+/ \mathrm{p}, \mathrm{s}$ & $+/ \mathrm{p}, \mathrm{s}$ & $+/ \mathrm{p}, \mathrm{s}$ & $+/ \mathrm{p}$ & $+/ \mathrm{p}$ & $+/ \mathrm{p}, \mathrm{s}$ & $+/ \mathrm{s}$ & $+/ \mathrm{p}, \mathrm{s}$ \\
\hline PMWS & $+/+$ & $-1-$ & $+/+$ & $+/+$ & $+/+$ & $+/+$ & $+/+$ & $+/+$ & $-1-$ & $+/+$ & $+/+$ & $+/+$ & $+/+$ & $+/+$ \\
\hline
\end{tabular}

*: Disease or lesions/the antigens.

-: Non-observed or non-detected.

+: Observed or detected (p: immunopositive PRRSV antigen, s: immunopositive Salmonella serovar O7 group).

§: Pigs which Salmonella Choleraesuis was isolated from lung and/or other tissues/organs including the ileum.

a): In the liver.

b): In residual abscesses of the lung.

c): in the contents of the ileum and colon.

antigens were detected in the degenerated cells which were phagocytosed with histocytic cells or giant cells in the nodules analogous to "paratyphoid" nodule (Fig. 1b). In the lung, many positive antigens to PCV2 were also detected in the cytoplasm of histocytic cells, infiltrated peripheral bronchi and alveolar epithelial cells. Positive antigens to PRRSV were detected in alveolar epithelial cells of twelve lungs $(85.7 \%)$. Furthermore, positive antigens to Salmonella serovar $\mathrm{O} 7$ group antigens were detected in infiltrating neutrophils in bronchiolar and alveolar lumens, and were also distributed in suppurative necrotising bronchopneumonia and in the residual abscesses. In liver sections, immunolabelling of bacterial cells was observed in the necrotic foci and in Kupffer cells. In the ileum and colon, the antigen was immunodetected in the infiltrated neutrophils in lamina propria of one $(14.2 \%)$ case.

$S$. Choleraesuis was randomly isolated from the organs of $8(57.1 \%)$ of the 14 diseased pigs (Table 1$)$. Of the organs examined, the bacteria were isolated from two parenchymal organs and the ileum. $S$. Choleraesuis isolation rates from the liver $(75.0 \%)$ and lung $(62.5 \%)$ were significantly higher than ileum (25.0\%). Other isolates were Actinobacillus pleuropneumoniae (21.4\%), Pasteurella multocida (14.3\%) and Streptococcus suis (7.1\%) from the lungs, besides Haemophilus parasuis $(7.1 \%)$ from one heart.

PCV2 genome was randomly detected in the lymphoid tissues and/or parenchymal organs obtained from all farms, except for one pig from farm C.

$S$. Choleraesuis infection has been ranked as one of the causative agents of infectious diseases of pigs, characterized by a tendency of opportunistic infections, as it has often been found concurrently with classical swine fever [23]. However, in recent years, the infection has been well known as a causative agent of septicemic disease in swine and has been the most frequent Salmonella serotype recovered from swine salmonellosis in North America [25] and Taiwan [10].

In Japan, between 1989 and 2004 Animal Hygiene Weekly (published periodically by the Animal Health and Animal Products Safety Division of the Ministry of Agriculture, 
Forestry and Fisheries), reported that in 1989 only two farms became ill with salmonellosis but the reports of the disease gradually increased from 1994 to 2004 when 68 farms were recorded with the disease. The weekly reports included several Salmonella serotypes in addition to $S$. Choleraesuis. Nevertheless, since the beginning of the 1990's, salmonellosis caused by $S$. Choleraesuis has been found in many districts in Japan $[11,12,23,30]$ and it is quite likely that many cases in the statistics has been caused by $S$. Choleraesuis.

Schwartz [24] suggested that several kinds of stressors were associated with immunosuppression in pigs, including viral infections, and these may potentiate or exacerbate clinical outbreaks of salmonellosis. Stevenson et al. [29] reported that infection concurrent with PRRSV contributed to an increase in the mortality rate of postweaning pigs due to $S$. Choleraesuis in natural cases. Furthermore, Wills et al. [31] confirmed experimentally that PRRSV and an additional injection of dexamethasone exacerbated clinical signs of $S$. Choleraesuis infection in pigs. Thus, differences in the disease conditions attributed to PRRS may be explained, at least in part, by the interactions of PRRSV-infection with the environment surrounding the pig ranches and other infectious agents [31].

In the present investigation, Twelve $(85.7 \%)$ of the 14 diseased pigs with clinically wasting and respiratory disorder were recorded as PMWS lesions. PCV2 and PRRSV as co-factors can result in more severe PMWS in conventional pigs under experimental conditions [21]. In Japan, there have been also many reports on the concurrent infection of PRRSV in PMWS affected pigs [12, 16, 22]. In the present investigation, PRRSV antigens were immunolabeled in the interstitial pneumonia in ten $(83.3 \%)$ of 12 PMWS affected pigs.

One of the important pathological findings of PMWS is a characteristically variable degree of lymphocyte depletion with loss of the follicular architecture [28] in the lymphatic tissues. The pathological findings strongly imply an immunosuppressive status in PMWS affected pigs. Thus, it is quite likely that PMWS is capable of suppressing the immune function sufficiently to induce secondary infections [26].

In the present cases, it may be true that septiceamic salmonellosis caused by $S$. Choleraesuis occurred in $9(64.3 \%)$ of the 14 diseased pigs (table 1). Of this population, 7 (77.8\%) were sick with PMWS, PRRSV antigens were detected in $5(71.4 \%)$ lungs of the 7 salmonellosis and PMWS affected pigs. These results show that the number of pigs with salmonellosis and PMWS concurrently infected with PRRSV (55.6\%) is much higher than those with salmonellosis and PMWS $(22.2 \%)$ or with salmonellosis and PPPRV (22.2\%).

It is known that the infectious route of $S$. Choleraesuis is respiratory and gastro-enteric tracts. In the present case, the organism and the Salmonella serovar group antigen were also isolated from the ileum and immunodetected in the ileum and colon in only one case. However, the present out- come was not conspicuous as the experimental gastric infection of the organism reported by Reed et al. [17]. It is presumably $S$. Choleraesuis infection might have established already from respiratory tract before the infection from the gastro-enteric tract.

PCV2 immunolabelling in the apoptotic hepatocytes has been already reported by Rosell et al. [20] and Hirai et al. [8]. PCV2 antigens were also found in the degenerated cells which were phagocytosed by histocytes or multinuclear giant cells in the nodules, being analogous to "paratyphoid" nodules in one case. "Paratyphoid" nodules are not recognized as granulomas with foreign body type giant cells, because they are characterized by only a small group of histocytes and necrotic hepatocytes. However, it might be difficult to differentiate the present lesions from "paratyphoid" nodules, when PMWS affected pigs are concurrently infected with $S$. Choleraesuis. Okuda et al. [15] has reported a case with hepatic lesions characterized by infiltration of multinucleated giant cells associated with PCV2 in a piglet. In addition, the present hepatic lesions was akin to the stage II of PCV2 infected liver, described as lymphohistocytic inflammation of the portal tracts accompanied by clusters of mononuclear inflammatory cells in the parenchyma by Rosell et al. [19]. Thus, it seems that the nodules analogous to "paratyphoid" nodules are rarely seen in advanced hepatic lesions in PMWS affected pigs with $S$. Choleraesuis infection. Thus, since the septicemic lesions [17] due to $S$. Choleraesuis are akin to interstitial pneumonia caused by PRRSV or PCV2 and the hepatic lesions are similar to those by PCV2, it is necessary to use the immunohistochemical technique to obtain an exact diagnosis.

PMWS has been ranked as the latest emerging infectious disease in pigs, since it was found in Canada by Harding [7]. It is possible that many of the pathological features ascribed to PRRSV could be attributable to infection with PCV2. Moreover, the diseased pigs which present clinical wasting syndrome could be recognized as PMWS [3]. We conducted the pathological and immunohistochemical investigations on one case of PRRS in 1993, and the results were reported by Hirose et al. [9]. Characteristic PMWS lesions revealed in the lymphoid organs, and PCV2 antigens were immunolabeled in the histocytic cells in the kidney (unpublished data). We surmise that, in the 1990's, it was possible that many cases of salmonellosis with concurrent infection of PRRSV were diagnosed as PMWS.

In the present investigation, the occurrences of salmonellosis in PMWS affected pigs with concurrent infection of PRRSV were very similar to the experimental cases reported by Wills et al. [31]. Thus, it might be possible that immunosuppressive status in PMWS may play a role of the aggravating agents in swine salmonellosis, when PRRSV infection follows to PMWS. On the other hand, clinically normal pigs but being in a carrier state may create troublesome problems for the control of the disease. When carrier pigs are brought from a farm contaminated with $S$. Choleraesuis to the second farm where there have been PMWS and/or PRRSV, it is quite likely that the infection will result 
in a heavy mortality in those pigs without immunity to $S$. Choleraesuis as carrier pigs shed $S$. Choleraesuis in faeces at low levels [4].

ACKNOWLEDGEMENTS. The authors would like to thank Dr M. Haritni, National Institute Animal Health, for kindly providing Biotinylated anti-PCV porcine serum and for cooperative immunostaining on one case of PRRS in 1993, which had been already reported by Hirose et al. [9].

\section{REFERENCES}

1. Allan, G. M., McNeilly, F., Ellis, J., Krakowka, S., Meehan, B., McNair, I., Walker, I. and Kennedy, S. 2000. Arch. Virol. 145: 2421-2429.

2. Ellis, J. A., Bratanich, A., Clark, E. G., Allan, G., Meehan, B., Haines, D. M., Harding, J., West, K. H., Krakowka, S., Konoby, C., Haard, L., Martin, K. and McNeilly, F. 2000. J. Vet. Diagn. Invest. 12: 21-27.

3. Ellis, J. A., Krakowka, S., Allan, G., Clark, E.and Kennedy, S. 1999. Vet. Pathol. 36: 262-265.

4. Gray, J., Fedorka-Cray, P. J., Stabel, T. J. and Ackermann, M. R. 1995. Vet. Microbiol. 47: 43-59.

5. Hamel, A. L., Lin, L. L. and Nayar, G. P. S. 1998. J. Virol. 72: 5262-5267.

6. Harms, P. A., Sorden, S. D., Halbur, P. G., Bolin, S. R., Lager, K. M., Morozov, I. and Paul, S. 2001. Vet. Pathol. 38: 528539.

7. Harding, J. C. 1996. p. 21. In: Proc. of the Swine Prac.

8. Hirai, T., Nunoya, T., Ihara, T., Kusanagi, K., Kato, T. and Shibuya, K. 2003. J. Vet. Med. Sci. 65: 1041-1045.

9. Hirose, O., Ono, M., Shibata, I, Kageyama, K., Kudoh, H., Yoshizawa, Nishimura, S., M., Samegai, Y. and Sakano, T. 1996. J. Jpn. Vet. Assoc. 49: 232-236 (in Japanese with English summary).

10. Hus, F. S., Chueck, L. L. and Shen, Y. M. 1983. Chin. J. Microbiol. Immunol. 16: 283-290.

11. Imafuji, T., Nakazaki, I., Tsuruta, R., Hagie, T., Kamimiyata, M., Furukawa, M. and Kitano, Y. 1993. J. Jpn. Vet. Assoc. 46: 909-912 (in Japanese with English summary).

12. Ito, N., Matsumoto, A., Takato, T., Inui, T., Nakane, T. and Murakami, S. 2003. J. Vet. Med. (Tokyo) 56: 185-190 (in Japanese with English summary).

13. Kennedy, S., Moffett, D. McNeilly, F., Meehan, B., Ellis, J., Krakowka, S. and Allan, G. M. 2000. J. Comp. Pathol. 122: 924.
14. Krakowka, S., Ellis, J. A., McNeilly, F. and Allan, G. 2000. Vet. Pathol. 37: 254-263.

15. Okuda, K., Shiraishi, M., Yoshimura, N., Tada, K., Fukutomi, T., Kayahara, Y., Haritani, M., Kawashima, K. and Murakami, Y. 2003. J. Jpn. Vet. Assoc. 54: 181-184 (in Japanese with English summary).

16. Onuki, A., Abe, K., Togashi, K., Kawashima, K., Taneichi, A. and Tsunemitsu, H. 1999. J. Vet. Med. Sci. 61: 1119-1123.

17. Reed, W. M., Olander, H. J. and Thacker, H. L. 1986. Am. J. Vet. Res. 47: 75-83.

18. Rodríguez-Arrioja, G. M., Segalés, J., Rosell, C., Quintana, J., Ayllón, S., Camprodón, A. and Domingo, M. 1999. Vet. Rec. 144: $152-153$.

19. Rosell, C., Segalés, J., Plana-Durán, J., Balasch, M., Rodríguez-Arrioja, G. M., Kennedy, S., Allan, G. M., McNeilly, F., Latimer, K. S. and Domingo, M. 1999. J. Comp. Pathol. 120: 59-78.

20. Rosell, C., Segalés, J. and Domingo, M. 2000. Vet. Pathol. 37: 687-692.

21. Rovira, A., Balasch, M., Segalés, J., García, L., Plana-Durán, J., Rosell, C., Ellerbrok, H., Mankertz, A. and Domingo, M. 2002. J. Virol. 76: 3232-3239.

22. Sato, K., Shibahara, T., Ishikawa, Y., Kondo, H., Kubo, M. and Dakota, K. 2000. J. Vet. Med. Sci. 62: 637-633.

23. Sato, Y., 1998. Proc. Jpn. Pig Vet. Soc. 33: 12-14 (in Japanese).

24. Schwartz, K. 1991. Comp. Contin. Educ. Pract. Vet. 13: 139147.

25. Schwartz, K. 1999. pp. 535-551. In: Diseases of Wine. 8 th ed. (Straw, B. E., D’Allaire, S., William, L. M. and Taylor D. eds.), Iowa State University Press, Ames AI, U.S.A.

26. Segalés, J., Domingo, M., Chianini, F., Majó, N., Domínguez, J., Darwich, L. and Mateu, E. 2004. Vet. Microbiol. 98: 151158.

27. Segalés, J., Calsamiglia, M., Rosell, C., Soler, M., Maldonado, J., Martín, M. and Domingo, M. 2002. Vet. Microbiol. 85: 23 30 .

28. Segalés, J., Rosell, C. and Domingo, M. 2004. Vet. Microbiol. 98: $137-149$.

29. Stevenson, G. W., Van Alstine, W. G., Kanitz, C. L. and Keffaber, K. K. 1993. J. Vet. Diagn. Invest. 5: 432-434.

30. Tanaka, N., Udagawa, K. and Hikita, K. 1994. J. Jpn.Vet. Assoc. 47: 937-939 (in Japanese with English summary).

31. Wills, R. W., Gray, J. T., Fedorka-Cray, P. J., Fedorka-Cray, K-J., Ladely, S. and Zimmerman, J. J. 2000. Vet. Microbiol. 71: $177-192$. 